\title{
BMJ Open Protocol for a within-trial economic evaluation of a psychoeducational intervention tailored to people at high risk of developing a second or subsequent melanoma
}

\author{
M Dieng, ${ }^{1}$ A E Cust, ${ }^{1,2} \mathrm{~N}$ A Kasparian, ${ }^{3}$ P Butow, ${ }^{4}$ D S J Costa,${ }^{4,5}$ S W Menzies, $, 6,7$ \\ G J Mann, ${ }^{2,8} \mathrm{R}$ L Morton ${ }^{9}$
}

To cite: Dieng M, Cust $A E$, Kasparian NA, et al. Protocol for a within-trial economic evaluation of a

psychoeducational

intervention tailored to people at high risk of developing a second or subsequent melanoma. BMJ Open 2016;6:e12153.

doi:10.1136/bmjopen-2016012153

- Prepublication history for this paper is available online. To view these files please visit the journal online (http://dx.doi.org/10.1136/ bmjopen-2016-012153).

Received 5 April 2016 Revised 5 August 2016 Accepted 14 September 2016

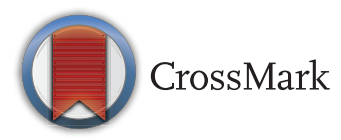

For numbered affiliations see end of article.

Correspondence to Mbathio Dieng; mbathio. dieng@sydney.edu.au

\section{ABSTRACT}

Introduction: Psychological support programmes are not currently funded for people with a history of melanoma. A major barrier to the implementation of effective psychological interventions in routine clinical care is a lack of cost-effectiveness data. This paper describes the planned economic evaluation alongside a randomised controlled trial of a psychoeducational intervention for people with a history of melanoma who are at high risk of developing new primary disease.

Method and analysis: The economic evaluation is a within-trial analysis to evaluate the incremental costs and health outcomes of a psychoeducational intervention compared to usual care from the perspective of the Australian healthcare system. Costeffectiveness and cost-utility analyses will be conducted, providing estimates of the cost to reduce fear of melanoma recurrence and the cost per quality-adjusted life-year (QALY) gained. Fear of melanoma recurrence will be measured using the Fear of Cancer Recurrence Inventory and preference-based quality of life measured using the Assessment of Quality of Life-8 Dimensions (AQoL-8D) instrument. The AQoL-8D will provide utilities for estimation of QALYs in the cost-utility analysis. Unit costs of health services and medicines will be taken from the Medicare Benefits Schedule and the Pharmaceutical Benefits Scheme national databases. Health outcomes, and health service and medication use will be collected at baseline, 6 and 12 months follow-up. The within-trial analysis will be conducted at 12 months, consistent with the end point of the trial.

Ethics and dissemination: Approval to conduct the study was granted by the Sydney Local Health District (RPAH zone) Ethics Review Committee (X13-0065 and HREC/13/RPAH/86), the Department of Health and Ageing Human Research Ethics Committee (21/2013), the University of Sydney Human Research Ethics Committee (2013/595), and the Australian Institute of Health and Welfare Ethics Committee (E0 2013/4/58). Trial registration number: ACTRN12613000304730; Pre-results.
Strengths and limitations of this study

- We have performed a number of preliminary studies, including (1) a systematic review of the literature on economic evaluations of psychological interventions to inform cost drivers and (2) a pilot study of the intervention in three melanoma clinics to determine the feasibility and acceptability of self-reported health service use and cost-related data collection.

- The protocol follows recognised guidelines to design and report economic evaluations nested in a randomised controlled trial and will include individual level data, which include objective measures of health service and medicine use, obtained by linkage to national data collections, overcoming the limitations of self-report.

- In Australia, there is not an explicit willingness to pay threshold for reduction of fear of cancer recurrence in people with cancer, or an explicit willingness to pay threshold for a quality-adjusted life-year gained.

- The Fear of Cancer Recurrence Inventory clinical cut-off point has been validated in those with other cancers but not in people at high risk of developing second or subsequent melanomas.

- A further potential limitation is the short duration of the trial, with cost-effectiveness being calculated at 12 months.

\section{INTRODUCTION}

Research supports the value of psychological interventions to enhance psychological adjustment, mental health and quality of life of people with melanoma. ${ }^{1}$ In addition, guidelines recommend that psychological and psychoeducational interventions be offered as part of routine clinical care for people with melanoma. ${ }^{2}$ Currently, however, there are no funded psychological support programmes for melanoma survivors during 
their post-treatment follow-up. A major barrier to the implementation of these interventions in routine clinical care is a lack of data on their cost-effectiveness. In a recently published systematic review, we identified only eight economic evaluations of psychological interventions in cancer, and only one (a retrospective costeffectiveness analysis (CEA)) was carried out in the melanoma context. ${ }^{3}$

Given the increasingly limited funding for healthcare resources, it is vital that psychological interventions be assessed for their impact on health outcomes and value for money. To facilitate decision-making, it is important to consider whether implementing an intervention is worthwhile given the incremental costs and incremental benefits that may be generated. The additional cost per health outcome considered 'worthwhile' is based on willingness to pay thresholds as well as other key criteria, usually defined by government decision makers. An intervention that is cost-effective has an incremental cost-effectiveness ratio (ICER) lower than the funders' willingness to pay threshold. ${ }^{4}$ An alternative approach to using the ICER is the net benefit approach, where a cost-effective intervention is the one with the highest net benefit. $^{45}$

Recommended best practice for economic evaluations of healthcare interventions is that they are conducted prospectively alongside a randomised controlled trial (RCT) ${ }^{6}$ We are conducting a RCT of a psychoeducational intervention for people with a history of melanoma who are at high risk of developing new primary disease (ie, a second or subsequent melanoma). To heighten the transparency of our methods and results and to enable a detailed description to be provided, we describe in this paper the protocol for a within-trial economic evaluation of the psychoeducational intervention based on 12-month follow-up data. The economic question to be addressed is whether the psychoeducational intervention is cost-effective from a health system perspective, compared to usual care, in reducing the proportion of participants who report fear of melanoma recurrence at a level warranting clinical intervention, and cost-effective in terms of quality-adjusted survival.

\section{METHODS AND ANALYSIS \\ Design}

Full details of the RCT protocol have been published. ${ }^{7}$ In brief, the Melanoma Care Intervention Study was a two-arm RCT designed for people with a history of melanoma who are at high risk of developing new primary disease and who were attending one of three high-risk melanoma clinics across New South Wales (NSW), Australia. People attending the clinics have had a melanoma and carry an additional risk factor, either multiple primary invasive melanomas, dysplastic nevus syndrome (ie, many atypical moles) or a strong family history; alternatively they could carry a high-penetrance mutation in
CDKN2A or CDK4 (with or without a melanoma diagnosis). Recruitment for the trial was conducted between February 2014 and January 2015 at the Sydney Melanoma Diagnostic Centre, the Melanoma Institute Australia (both in metropolitan Sydney) and the Newcastle Skin Check Clinic, located in a regional, coastal area. Randomisation was performed, ensuring allocation concealment, using the telephone randomisation service at the Australian National Health and Medical Research Council (NHMRC) Clinical Trials Centre, The University of Sydney. Recruited participants were assigned an identification code and randomised using minimisation, stratified by high-risk clinic site.

The economic evaluation is a within-trial analysis to evaluate the additional costs and health outcomes of the psychoeducational intervention compared to usual care from the perspective of the Australian healthcare system. Economic evaluations are comparative analyses of alternative interventions in regards to costs, resource use and health outcomes. ${ }^{8}$ This protocol outlines the methods for an economic evaluation of a CEA and cost-utility analysis (CUA) to evaluate the cost per health outcome gained. The difference between the two analyses is the measure of effectiveness used. With CEA, costs are expressed in monetary units (eg, dollars) and effects in clinical outcome units (eg, additional people in whom the fear of melanoma recurrence-at a level warranting clinical intervention-is averted), while for CUA, costs are expressed in monetary units and effects in quality-adjusted life-years (QALYs) gained. The health system perspective considers costs and outcomes relevant to the Australian healthcare system. This was informed by a recent systematic review of economic evaluations of psychological and psychoeducational interventions in cancer, which reported that the main drivers of costs were direct medical costs. ${ }^{3}$

\section{Study population}

All trial participants will be included in the economic evaluation. Participants attending the high-risk melanoma clinics were eligible for the RCT if previously diagnosed with American Joint Committee on Cancer $(\mathrm{AJCC})^{9}$ melanoma stage 0 , I or II, were aged 18 years or older and with sufficient English language skills to complete all aspects of the study without the assistance of an interpreter. All participants gave informed consent to take part in the RCT, including consent to access their records from the national Medicare Benefits Schedule (MBS) and Pharmaceutical Benefits Scheme (PBS) databases.

\section{Intervention and comparator}

The intervention was designed to address the gap in psychological support for people affected by melanoma. The intervention was comprised of an evidence-based psychoeducational booklet, 'Melanoma: Questions and Answers', and three individually tailored, telephonebased psychotherapeutic sessions with a psychologist. 
The control group received usual care and were provided with the cancer council booklet 'Understanding Melanoma'. A summary of the intervention and comparator components is presented in table 1.

\section{Outcome measures}

Table 2 summarises the outcome measures and time of collection for the outcomes that will be used in the economic evaluation. Outcome data were collected using self-reported questionnaires by the trial participants at baseline (before randomisation), 6-month and 12-month follow-up.

Two economic outcomes will be calculated. First, the primary outcome of effectiveness for the CEA will be the proportion of participants who do not report fear of melanoma recurrence warranting clinical intervention, in the past month. Fear of new or recurrent melanoma was assessed using the Severity subscale of a modified (ie, melanoma-specific) version of the 42-item Fear of Cancer Recurrence Inventory (FCRI).$^{10}$ The FCRI was categorised into two levels: FCRI Severity scores within the 'normal' range (scores ranging from 1 to 12) and FCRI Severity scores indicating a potential need for clinical intervention (scores ranging from 13 to 36$).{ }^{11} \mathrm{~A}$ higher score is indicative of greater fear of cancer recurrence.

Second, for the CUA, the Assessment of Quality of Life-8 Dimensions (AQoL-8D) will be used to measure health-related quality of life and provides utilities for estimation of QALYs. ${ }^{12}{ }^{13}$ The AQoL-8D is a preferencebased measure, designed to record health-related quality of life in a format amenable to economic evaluation. It is specifically developed for interventions that aim to improve mental health, with norms for the Australian population by age and gender. It is sensitive to change after psychological interventions because it includes domains such as depression/anxiety, social function and self-esteem. The AQoL-8D is a 35-item health-related quality of life preference-based measure from which eight dimensions and two 'superdimensions' are derived. ${ }^{12}$ The total 35 -item score will be converted to a single utility score using the AQoL-8D algorithm. ${ }^{13}$

\section{Resource use and costs}

For the within-trial analysis, only costs and effects that accumulate within the trial up to 12 months will be included. Costs will be identified and valued as all melanoma-related direct costs, hospital visits, hospital admissions, doctor visits, allied health services use (eg, psychologist, genetic counsellor, dietician) and intervention costs. Table 3 presents an overview of the resource use and cost measures to be used in the economic evaluation; this includes costs hypothesised to differ between intervention and control groups, costs associated with intervention development and delivery, health services and medication costs.

Costs associated with intervention development will include development and pilot testing of the psychoeducational booklet, 'Melanoma: Questions and Answers', as well as development of the intervention manual, psychologist recruitment and training, and pilot testing of the intervention. Human resource records and intervention development team records will be used for the valuation of these costs.

The trial-associated costs will take into account the salaries and administrative costs associated with coordinating the trial, production of the trial materials, postage, psychologist salaries, weekly clinical supervision costs, the recording material and telephone use (number and duration of calls). We will not include the cost of the routinely issued Cancer Council booklet, 'Understanding Melanoma', because this is being offered to both groups; hence, the cost is identical in both groups. Research team and psychologists' records relating to intervention delivery and the human resources records will be used to value the costs.

All resource use related to the psychoeducational intervention, including the use of mental health services and any direct costs related to melanoma diagnosis, treatment or risk management, including medicines, will be quantified. Unit costs for health services and medication use will be estimated from linkage to the national Medicare (MBS) and Pharmaceutical (PBS) databases. Other service use not recorded in MBS or PBS (eg, hospital admission, private psychology services, use of complementary therapies) will be based on participant self-report, collected at baseline, 6-month and 12-month follow-up. Participants' total health service use cost will be the aggregation from the number of services used by the unit cost for the service.

All resources will be valued in 2014 Australian dollars. No discounting will be applied as follow-up is over 1 year only. The cost of the booklet development in 2013 will be inflated to the current reference year.

\section{Statistical analysis}

Analyses of the main study outcomes will be carried out according to intention to treat. ${ }^{6}$ For the economic evaluation, all statistical tests and CIs will be two sided, with statistical significance inferred at the 0.05 level. Potential differences in costs and health outcomes between the two groups and the 95\% CIs will be presented. Since costs and QALYs are usually non-normally distributed, ${ }^{14}$ we will use generalised linear models ${ }^{15}$ for adjustment of baseline covariates. The effect of the intervention will be estimated using a linear mixed model with random intercept, including time as a repeated categorical variable and adjusting for baseline scores (ie, baseline outcome scores will be included as covariates in the model). Baseline covariates will include age, gender, family income, education level and marital status. CIs for the ICERs will be calculated using a non-parametric bootstrap procedure. Statistical analysis will be performed using SAS V.9.3 (SAS Institute, Cary, North Carolina, USA) and Excel (Microsoft Corp., Redmond, Washington, USA). 
Table 1 Summary of the intervention and comparator components

Component
Full dermatological
appointment at the high-risk
clinic

Psychoeducational booklet, 'Melanoma: Questions and Answers'

Cancer Council booklet, 'Understanding Melanoma'

Psychotherapeutic Session 1

Psychotherapeutic Session 2

Psychotherapeutic Session 3

Description
Full dermatological appointments at the
high-risk clinics designed to screen
patients using different techniques,
including dermoscopy, digital
dermoscopy monitoring and total body
photography

A 76-page, full-colour, evidence-based, psychoeducational booklet comprised of seven modules and a series of tailored resources. The booklet features comprehensive information on a range of topics, including melanoma types and risk factors, clinical care for people affected by melanoma, tools for skin self-examination and doctor-patient communication, psychological aspects associated with melanoma, including living with fear of new or recurrent melanoma, coping responses, tools for keeping track of one's care, and pathways to further information and support

Easy-to-read information about melanoma diagnosis, treatment, and emotional and practical issues. The booklet was sent to participants 4 weeks prior to their next full dermatological appointment

Scheduled 1 week prior to patients' 6-monthly full dermatological appointment. Session 1 featured a psychological assessment, including discussion of participants' background, experiences of melanoma and clinical care, other health-related experiences, information and support needs, and goals, hopes and wishes for the intervention

Session 2 included exploration of participants' experience of his or her recent high-risk melanoma clinic appointment and its outcomes, the clinical care received, review of previous session and any difficulties experienced, participants' unmet information and support needs, and information and referral for addressing unmet needs, when appropriate

Session 3 comprised: review of previous session and any difficulties experienced since the session, discussion of the degree to which unmet needs have been addressed, discussion of new strategies to address potential future difficulties or concerns, exploration of relevant services and resources in the booklet and other sources and facilitation of referral for ongoing psychological care, if indicated
Four weeks prior to 6-monthly full dermatological appointment

Four weeks prior to 6-monthly full dermatological appointment

One week prior to

6-monthly full dermatological appointment

One week after 6-monthly full dermatological appointment

Three weeks after 6-monthly full dermatological appointment

Usual care

Every 6 months

At the end of the trial

Four weeks prior to

6-monthly full

dermatological

appointment

Never

Never

Never 
Table 2 Overview of health outcomes measured

\begin{tabular}{|c|c|c|c|}
\hline Health outcome & Means of collection & Timing of collection & Source of data \\
\hline $\begin{array}{l}\text { Fear of cancer } \\
\text { recurrence (severity } \\
\text { score) }\end{array}$ & $\begin{array}{l}\text { FCRI administered } \\
\text { via self-report } \\
\text { questionnaire }\end{array}$ & $\begin{array}{l}\text { Baseline prior to randomisation } \\
6 \text {-month follow-up } \\
\text { 12-month follow-up }\end{array}$ & $\begin{array}{l}\text { Patient-reported } \\
\text { outcomes }\end{array}$ \\
\hline $\begin{array}{l}\text { Health-related quality of } \\
\text { life }\end{array}$ & $\begin{array}{l}\text { AQoL-8D } \\
\text { administered via } \\
\text { self-report } \\
\text { questionnaire }\end{array}$ & $\begin{array}{l}\text { Baseline prior to randomisation } \\
6 \text {-month follow-up } \\
\text { 12-month follow-up }\end{array}$ & $\begin{array}{l}\text { Patient-reported } \\
\text { outcomes }\end{array}$ \\
\hline
\end{tabular}

Table 3 Overview of resource use measures

\begin{tabular}{|c|c|c|c|}
\hline Item of resource use & Unit & Unit costs & Source of data \\
\hline \multicolumn{4}{|l|}{ Intervention } \\
\hline Booklet development & Hour research time & AUD/hour & Grant budget \\
\hline \multicolumn{4}{|l|}{ Pilot testing } \\
\hline Booklet design & Hour Graphic design & AUD/hour & Designer invoice \\
\hline Booklet printing & Booklet & AUD/booklet & Printing invoice \\
\hline Questionnaire printing & Questionnaire & AUD/survey & Printing invoice \\
\hline Postage & Reply paid envelope & AUD/envelope & Trial records \\
\hline Intervention manual development & Therapist hour & AUD/hour & University HR \\
\hline Psychologist recruitment & Advertisement & AUD/advert & APS invoice \\
\hline Psychologist training & Hour of training & AUD/hour & University HR \\
\hline Weekly clinical supervision & Hour of supervision & AUD/hour & Trial records \\
\hline \multicolumn{4}{|l|}{ Trial-related costs } \\
\hline \multirow[t]{2}{*}{ Trial coordination } & Hour PhD student & AUD/hour & University HR \\
\hline & Hour RA & AUD/hour & University HR \\
\hline Psychologist salary & Hour psychologist & AUD/hour & University HR \\
\hline Booklets printing & Booklet & AUD/booklet & University HR \\
\hline Questionnaires printing & Questionnaire & AUD/survey & Printing invoice \\
\hline Telephone counselling & Minutes telephone call & $\mathrm{AUD} / \mathrm{min}$ & Provider rates \\
\hline Recording materials & Recorder & AUD/recorder & Trial records \\
\hline Stamps & Set & AUD/set & Trial records \\
\hline Envelopes & Box & AUD/box & Trial records \\
\hline \multicolumn{4}{|l|}{ Health services use } \\
\hline Doctors' visits & Mean number of hours of visit & $85 \%$ scheduled fee in AUD & Medicare Australia \\
\hline \multicolumn{4}{|l|}{ Diagnostic testing } \\
\hline \multicolumn{4}{|l|}{ Psychologist visit } \\
\hline \multicolumn{4}{|l|}{ Psychiatrist visit } \\
\hline \multicolumn{4}{|l|}{ Social worker } \\
\hline \multicolumn{4}{|l|}{ Counselling service } \\
\hline \multicolumn{4}{|l|}{ Complementary therapies } \\
\hline \multicolumn{4}{|l|}{ Other allied health } \\
\hline \multicolumn{4}{|l|}{ Medication use } \\
\hline Prescribed medications & Dose & PBS fee & Medicare Australia \\
\hline
\end{tabular}

\section{Sample size}

The sample size was based on the main study clinical end points, not the expected difference in costeffectiveness. Sample size calculations were based on $80 \%$ power and a two-sided $\alpha=0.05$ test. Since there was not a validated, accepted estimate of the minimal clinically important difference for the Fear of Cancer Recurrence Inventory Severity subscale, we based the sample size calculation on a standardised mean difference (Cohen's d) of 0.5, which is a moderate effect size and is applicable to a wide variety of patient-reported outcomes. ${ }^{16}$ Based on previous research (not yet published), the SD was estimated as 29; thus, the expected mean difference in the FCRI scale was 14.5. Using these values, and after taking into account the maximum expected attrition rate of $20 \%$, the sample size required 
was 77 per group. Sample sizes based on economic outcomes are usually large in order to detect statistically significant differences due to the large variability in the use of healthcare resources and cost measures. ${ }^{17}$ It was therefore not feasible for our trial to be based on the economic end point because of the relatively limited number of patients attending the high-risk melanoma clinics. To overcome this limitation in the evaluation, we will plot a cost-effectiveness acceptability curve (CEAC). The CEAC is a graphical representation of the quantification of the uncertainty around the expected costeffectiveness, at different willingness to pay levels. ${ }^{5}$

\section{CEA and CUA}

The economic evaluation will use patient-level data on resource use and effects within the clinical trial period to assess the cost-effectiveness of the psychoeducational intervention compared to the usual care. Total and average costs for the intervention and control groups and the average cost per participant will be reported in disaggregated and aggregated formats. Total and mean outcomes (fear of cancer recurrence severity and quality-adjusted survival) will be reported for the intervention and usual care groups. For fear of cancer recurrence severity and QALYs, we will adjust for baseline values. ${ }^{18}$ ICER will be calculated and reported. Results of the CEA will be expressed as the incremental cost per additional person without a FCRI Severity subscale score above the clinical cut-off (13). Results of the CUA will be expressed as the incremental cost per QALY gained in the intervention group compared with the usual care group. ${ }^{4}$ Results will be plotted on a costeffectiveness plane.

The cost per person without a fear of melanoma recurrence at a level warranting clinical intervention will allow us to compare the cost-effectiveness results with other similar psychoeducational interventions in melanoma. The cost per QALY will be useful for comparison of cost-effectiveness with other cancers and diseases because QALYs are not disease specific. ${ }^{17}$ Reporting of the study design, methods and results will follow the Consolidated Health Economic Evaluation Reporting Standards (CHEERS) statement. ${ }^{19}$

\section{Missing data}

Mean imputation will be used to handle missing baseline values. Multiple imputation approaches will be used to handle missing outcomes data (eg, FCRI Severity scores). For AQoL-8D scores, we will follow the developer's guideline for missing data; that is, missing data will be handled by imputing values within each dimension. If more item responses in the dimensions are missing, the observations will be dropped and there will not be a dimension score or instrument score for the individual. $^{12}$ To address potential biases due to incomplete follow-up, we will use multiple imputation approaches to replace cost values that are missing at random or missing completely at random. ${ }^{142021}$ Since cost data are unlikely to be normally distributed, ${ }^{14}$ we will use the multiple imputation chained equations approach to impute missing cost data. Costs will be imputed at the total cost level. ${ }^{14}$

\section{Sensitivity analysis}

Bootstrapping will be used to estimate a distribution around costs and health outcomes, and to estimate the CIs around the ICER. Several one-way sensitivity analyses will be carried out. First, to address the uncertainty around the ICER relating to the external validity, we will carry out sensitivity analyses on the most important cost drivers to assess the impact of protocol-driven healthcare use. Second, total cost will be calculated with and without the cost of intervention development to ascertain whether an increased cost in the intervention arm could be explained by the cost of the intervention. Third, regression analyses will be carried out to ascertain more efficient estimates of trial-wide treatment costeffectiveness and allow the estimation of costeffectiveness relating to subgroups based on patient characteristics. ${ }^{22}$ We have collected several patient-level variables that will be used as covariates (eg, age, gender, education level, income level and previous clinical history) in a regression model. In addition, one-way sensitivity analyses will be conducted around the probability that the psychoeducational intervention is cost-effective across a range of values, and a CEAC $^{23}$ will be plotted.

\section{Ethics approvals and trial registration}

Approval to conduct the study was granted by the Sydney Local Health District (RPAH zone) Ethics Review Committee (13 March 2013; X13-0065 and HREC/13/RPAH/86), the Department of Health and Ageing Human Research Ethics Committee (12 August 2013), and the Australian Institute of Health and Welfare Ethics Committee (26 November 2013; EO 2013/4/58) for Medicare linkage. The trial was registered with the Australian and New Zealand Clinical Trials Registry on 19 March 2013 (Registration Number: ACTRN12613000304730). The RCT protocol has been endorsed by the Australia and New Zealand Melanoma Trials Group (ANZMTG) and by the Scientific Advisory Committee of the Psycho-oncology Co-operative Research Group (PoCoG).

\section{DISCUSSION}

To inform the implementation of interventions in clinical practice, guidelines recommend providing evidence of value for money; ${ }^{6}$ however, there are a limited number of economic evaluations alongside clinical trials available in the psycho-oncology literature. ${ }^{324}$ For transparency of reporting and to enable a detailed description of the economic evaluation methods to be provided, this paper presents the protocol of a withintrial economic evaluation aiming to assess the costeffectiveness of a psychoeducational intervention developed for people with a history of melanoma at high risk 
of developing new primary disease. This study will provide the first prospective, trial-based evidence on the healthcare costs associated with psychoeducational support for people with melanoma.

This study has several strengths. First, in preparation for the economic evaluation of the intervention, we have performed a number of preliminary studies, including (1) a systematic review of the literature on economic evaluations of psychological interventions to inform cost drivers and (2) a pilot study of the intervention in three melanoma clinics to determine the feasibility and acceptability of self-reported health service use and cost-related data. Second, this protocol follows recognised guidelines to design and report on economic evaluations nested in a RCT. $^{6}$ In addition, the economic evaluation will include individual-level data, which are preferable for economic evaluations. ${ }^{6}$ Importantly, these individual-level data include objective measures of health service and medicine use, obtained by linkage to national data collections, overcoming the limitations of self-report. ${ }^{25}$ Reliable economic evaluations are crucial to shape healthcare policy, in particular when the possibility of bias in economic evidence has been minimised by randomisation. ${ }^{26}$ Economic evaluations of psychological and psychoeducational programmes are also necessary to inform decision makers and to promote the funding and implementation of these interventions in clinical care. A review of the literature found a scarcity of cost-effectiveness studies related to psychological interventions in cancer in general and in melanoma in particular. ${ }^{3}$ The only previous study by Bares et $a t^{27}$ was a retrospective economic evaluation of a psychological intervention to alleviate emotional distress in people with melanoma. The authors found an ICER of $\$ 402.37$ for 1 point decrease of the Global Severity Index of the Brief Symptom Inventory scale for the usual care group, compared to an ICER of $\$ 7.66$ for 1 point decrease for the intervention group (usual care+cognitive-behavioural therapy). However, the study lacked methodological rigour in that the perspective chosen was not stated, not all costs and outcomes were properly identified and valued, and a sensitivity analysis was not conducted. ${ }^{3}$

A limitation of the proposed evaluation is that in Australia, there is not an explicit willingness to pay threshold for reduction of fear of cancer recurrence in people with cancer, or an explicit willingness to pay threshold for cost per QALY gained. However, an observation of the decisions of the Pharmaceutical Benefits Advisory Committee between 1994 and 2003 points to an 'arbitrary' threshold of \$A69 900 per QALY gained. ${ }^{28}$ In addition, the FCRI clinical cut-off point has been validated in those with other cancers but not in people at high risk of developing second or subsequent melanomas. Furthermore, the use of a clinical cut-point of 13 or above on the severity subscale of the FCRI has been proposed as the point at which people should be referred for a psychological assessment and potentially treatment. ${ }^{11}$ However, we would undertake a sensitivity analysis for the main economic outcome if future published cut-points were to differ markedly. Common to all cost-effectiveness analyses conducted alongside clinical studies, restrictive inclusion criteria and protocol-driven resource use among other factors could influence the external validity of the results. ${ }^{6}$ Therefore, we will conduct a range of sensitivity analyses around key variables (cost drivers, total cost calculated with and without the cost of the intervention development, patient characteristics) to address the uncertainty around the ICER relating to the external validity. A further potential limitation is the short duration of the trial, with costeffectiveness being calculated at 12 months.

\section{CONCLUSION}

The publication and peer-review of economic evaluation protocols alongside clinical trials is recommended to increase transparency and minimise bias. ${ }^{29}$ This study aims to contribute valuable evidence about the costeffectiveness of a psychoeducational intervention for people with a history of melanoma at high risk of developing new primary disease. The results of this study will be of immediate relevance for decision makers regarding implementation of this novel psychoeducational intervention for this patient group. In addition, if costeffectiveness is demonstrated, the multidisciplinary collaboration on this project, involving several melanoma clinics, psycho-oncology and melanoma trial groups and consumer groups, will facilitate future implementation.

\section{Author affiliations}

${ }^{1}$ Cancer Epidemiology and Prevention Research, Sydney School of Public Health, The University of Sydney, Sydney, New South Wales, Australia

${ }^{2}$ Melanoma Institute Australia, The University of Sydney, North Sydney, New South Wales, Australia

${ }^{3}$ Discipline of Paediatrics, School of Women's and Children's Health, UNSW Medicine, The University of New South Wales, Sydney, New South Wales, Australia

${ }^{4}$ Psycho-oncology Co-operative Research Group, School of Psychology, The University of Sydney, Sydney, New South Wales, Australia

${ }^{5}$ Pain Management Research Institute, University of Sydney at Royal North Shore Hospital, St Leonards, New South Wales, Australia

${ }^{6}$ Discipline of Dermatology, Sydney Medical School, The University of Sydney, Camperdown, New South Wales, Australia

${ }^{7}$ The Sydney Melanoma Diagnostic Centre, Royal Prince Alfred Hospital, Camperdown, New South Wales, Australia

${ }^{8}$ Centre for Cancer Research, Westmead Institute for Medical Research, The University of Sydney, Westmead, New South Wales, Australia

${ }^{9}$ NHMRC Clinical Trials Centre, The University of Sydney, Camperdown, New South Wales, Australia

Contributors MD, RLM, NAK, AEC, PB, GJM, SWM and DSJC contributed to the study design; MD, RLM and DSJC contributed to the statistical analyses; MD, NAK and AEC contributed to data acquisition; MD was responsible for the initial drafting of this manuscript; all authors contributed to the review of this manuscript and provided comments. All authors read and approved the final manuscript.

Funding This work was supported by a Cancer Institute NSW Program Grant for Excellence in Translational Research (GJM, SWM), and a Project Grant from beyondblue: the national depression initiative (NAK, ID 630575). MD was supported by a PhD scholarship funded through a Cancer Institute NSW fellowship to AEC, a Sydney Catalyst Top-Up Research Scholar Award and a 
Cancer Institute NSW Program Grant for Excellence in Translational Research (10/TPG/1-02; 2011-15) to GJM. AEC was supported by Career Development Fellowships from the National Health and Medical Research Council of Australia (NHMRC ID 1063593) and Cancer Institute NSW (15/CDF/1-14). NAK is the recipient of a Career Development Fellowship from the National Health and Medical Research Council of Australia (NHMRC ID 1049238). RLM was supported by a Sidney Sax Early Career Fellowship (NHMRC ID 1054216). PB was supported by a NHMRC Senior Principal Research Fellowship (ID 1022582). The RCT protocol has been endorsed by the Australia and New Zealand Melanoma Trials Group (ANZMTG) and by the Scientific Advisory Committee of the Psycho-oncology Co-operative Research Group (PoCoG).

Competing interests None declared.

Patient consent Obtained.

Ethics approval Sydney Local Health District (RPAH zone), Department of Health and Ageing Human Research, Australian Institute of Health and Welfare, University of Sydney Human Research Ethics Committee.

Provenance and peer review Not commissioned; externally peer reviewed.

Open Access This is an Open Access article distributed in accordance with the Creative Commons Attribution Non Commercial (CC BY-NC 4.0) license, which permits others to distribute, remix, adapt, build upon this work noncommercially, and license their derivative works on different terms, provided the original work is properly cited and the use is non-commercial. See: http:// creativecommons.org/licenses/by-nc/4.0/

\section{REFERENCES}

1. McLoone J, Menzies S, Meiser B, et al. Psycho-educational interventions for melanoma survivors: a systematic review. Psychooncology 2013;22:1444-56.

2. Australian Institute of Health and Welfare. Clinical practice quidelines for the management of melanoma in Australia and New Zealand. Wellington: Cancer Council Australia and Australian Cancer Network, Sydney and New Zealand Guidelines Group, 2008.

3. Dieng M, Cust AE, Kasparian NA, et al. Economic evaluations of psychosocial interventions in cancer: a systematic review. Psychooncology 2016. [Epub ahead of print 26 Jan 2016]. doi:10. 1002/pon.4075.

4. Drummond MF SM, Torrance GW, O'Brien BJ, et al. Methods for the economic evaluation of health care programmes. 3rd edn. Oxford University Press, 2005

5. Edlin R, McCabe C, Hulme C, et al. Cost effectiveness modelling for health technology assessment: a practical course. New York: Springer, 2015.

6. Ramsey S, Willke R, Briggs A, et al. Good research practices for cost-effectiveness analysis alongside clinical trials: the ISPOR RCT-CEA Task Force report. Value Health 2005;8:521-33.

7. Dieng M, Kasparian NA, Morton RL, et al. The Melanoma care study: protocol of a randomised controlled trial of a psycho-educational intervention for melanoma survivors at high risk of developing new primary disease. BMC Psychol 2015;3:23.

8. Hoch JS, Dewa CS. An introduction to economic evaluation: what's in a name? Can J Psychiatry 2005;50:159-66.
9. Balch CM, Gershenwald JE, Soong SJ, et al. Final version of 2009 AJCC melanoma staging and classification. J Clin Oncol 2009;27:6199-206.

10. Simard S, Savard J. Fear of Cancer Recurrence Inventory: development and initial validation of a multidimensional measure of fear of cancer recurrence. Support Care Cancer 2009;17:241-51.

11. Simard S, Savard J. Screening and comorbidity of clinical levels of fear of cancer recurrence. J Cancer Surviv 2015;9:481-91.

12. Richardson J, lezzi A, Khan MA, et al. Validity and reliability of the Assessment of Quality of Life (AQoL)-8D multi-attribute utility instrument. Patient 2014;7:85-96.

13. Richardson J, Sinha K, lezzi A, et al. Modelling utility weights for the Assessment of Quality of Life (AQoL)-8D. Qual Life Res 2014;23:2395-404

14. Faria R, Gomes M, Epstein D, et al. A guide to handling missing data in cost-effectiveness analysis conducted within randomised controlled trials. Pharmacoeconomics 2014;32:1157-70.

15. Briggs A, Sculpher M, Karl C. Decision modelling for health economic evaluation. New York: Oxford University Press, 2006.

16. Norman GR, Sloan JA, Wyrwich KW. Interpretation of changes in health-related quality of life: the remarkable universality of half a standard deviation. Med Care 2003;41:582-92.

17. Petrou S, Gray A. Economic evaluation alongside randomised controlled trials: design, conduct, analysis, and reporting. BMJ 2011;342:d1548.

18. Manca A, Hawkins N, Sculpher MJ. Estimating mean QALYs in trial-based cost-effectiveness analysis: the importance of controlling for baseline utility. Health Econ 2005;14:487-96.

19. Husereau D, Drummond M, Petrou S, et al. Consolidated Health Economic Evaluation Reporting Standards (CHEERS) statement. BMC Med 2013;11:1-6.

20. Briggs A, Clark T, Wolstenholme J, et al. Missing...presumed at random: cost-analysis of incomplete data. Health Econ 2003;12:377-92.

21. Schafer JL. Multiple imputation: a primer. Stat Methods Med Res 1999;8:3-15.

22. Hoch JS, Briggs AH, Willan AR. Something old, something new, something borrowed, something blue: a framework for the marriage of health econometrics and cost-effectiveness analysis. Health Econ 2003;11:415-30.

23. Fenwick E, O'Brien BJ, Briggs A. Cost-effectiveness acceptability curves-facts, fallacies and frequently asked questions. Health Econ 2004;13:405-15.

24. O'Sullivan AK, Thompson D, Drummond MF. Collection of health-economic data alongside clinical trials: is there a future for piggyback evaluations? Value Health 2005;8:67-79.

25. Glick HA, Doshi JA, Sonnad SS et al. Economic evaluation in clinical trials. 2nd edn. Oxford: Oxford University Press, 2015.

26. Gold MR, Siegel JE, Daniels N, et al. Cost-effectiveness in health and medicine: report of the panel on cost-effectiveness in health medicine. New York: Oxford University Press, 1996:283-5.

27. Bares CB, Trask PC, Schwartz SM. An exercise in cost-effectiveness analysis: treating emotional distress in melanoma patients. J Clin Psychol Med Settings 2002;9:193-200.

28. Henry DA, Hill SR, Harris A. Drug prices and value for money: The Australian Pharmaceutical Benefits Scheme. JAMA 2005;294:2630-2.

29. Godlee F. Publishing study protocols: making them visible will improve registration, reporting and recruitment. BMC News Views $2001 ; 2: 4$. 